\title{
$\epsilon$-Compatible Map and New Approach for Common Fixed Point Theorems in Partial Metric Space Endowed with Graph
}

\author{
Anuradha*, SeemaMehra \\ Department of Mathematics, Maharshi Dayanand University (M.D.U), India
}

Received January 14, 2020; Revised March 31, 2020; Accepted April 19, 2020

Copyright $\odot 2020$ by authors, all rights reserved. Authors agree that this article remains permanently open access under the terms of the Creative Commons Attribution License 4.0 International License

\begin{abstract}
In 2016, Muralisankar and Jeyabal introduced the concept of $\varepsilon$-Compatible maps and studied the set of common fixed points. They generalized the Banach contraction, Kannan contraction, Reich contraction and Bianchini type contraction to obtain some common fixed point theorems for $\varepsilon$ - Compatible mappings which don't involve the suitable containment of the ranges for the given mappings in the setting of metric spaces. Motivated by this new concept of mappings, we establish a new approach for some common fixed point theorems via $\epsilon$-compatible maps in context of complete partial metric space including a directed graph $\mathrm{G}=(\mathrm{V}, \mathrm{E})$. By the remarkable work of Jachymski in 2008, we extend the results obtained by Muralisankar and Jeyabal in 2016. In 2008, Jachymski obtained some important fixed point results introduced by Ran and Reurings (2004) using the languages of graph theory instead of partial order and gave an interesting approach in this direction. After that, his work is considered as a reference in this domain. Sometimes, there are some mappings which do not satisfy the contractive nature on whole set M(say) but these can be made contractive on some subset of $\mathrm{M}$ and this can be done by including graph as shown in our Example 2.6 which is provided to substantiate the validity of our results.
\end{abstract}

Keywords Common Fixed Point, Complete Partial Metric Space, $\epsilon$-Compatible Map, Directed Graph

MSC: 47H09; 47H10; 47E10

\section{Introduction and Preliminaries}

French mathematician Maurice Frechet in 1906 introduced the concept of metric space. By following the work of Frechet, several generalization of metric space came out by several authors and one of the generalization is partial metric space that introduced by Mathews [14] to obtain appropriate mathematical models in the theory of computation.

In the research of modern mathematics, the theory of fixed point is one of the most powerful tools. By the fixed point theorems, we mean that Theorems concerning the existence and properties of fixed point. The classical theorem formulated by Stephen Banach in 1922, in non-linear functional analysis which state that a contraction mapping on complete metric space (M,d) has a unique fixed point and also conveys the method how to compute the fixed point through iteration process. This famous Banach principle also gave birth to many other remarkable fixed point theorem such as Ciric (1974), Rhoadas (1996), Kirk (1983), Park (1998), Nulder (1969) and etc.

Study of common fixed point has been at the centre of high-spirited research activity. In 1976, Jungck [4] generalized the Banach contraction principle for more than one mapping to obtain common fixed point theorems for commuting mappings such that one of them is continuous. His theorem states that,

Theorem 1.1[4]. Let ( $\mathrm{M}, \mathrm{d})$ be a complete metric space and let $f$ and $g$ be commuting self-maps of $M$ satisfying the conditions:

i) $\mathrm{f} \mathrm{M} \subseteq \mathrm{g}_{\mathrm{g}} \mathrm{M}$

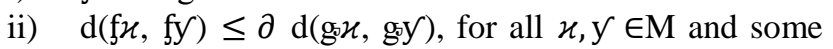
$\partial \in(0,1)$.

If $g_{s}$ is continuous then $f$ and $g_{s}$ have a unique common fixed point.

Onwards, Jungck and Rhoades [3] have introduced the concept of compatibility of maps which is weaker condition than weak commutativity and obtained some common fixed point results for these kind of mappings. Generally, common fixed point theorem involves conditions on commutativity, continuity, completeness and 
suitable containment of ranges of the involved mappings apart from an propitious contraction condition and investigators in this area are aimed at weakening one or more of these conditions. In this direction, one more step is taken out by other researchers to find common fixed point via $\varepsilon$-compatible map which doesn't involve suitable containment of ranges of the involved mappings.

In current trend of research, the study of fixed point theory involving graph inhabits/occupies a noteworthy place in many fields. In 2008, Jachymaski [9] was studied first the Banach Contraction Principle on a metric space with a graph. The results obtained byJachymaski generalize the many recent results of other researchers obtained in a partially ordered metric space. According to Jachymaski, using the language of graph theory instead of a partial ordering is more convenient. A lot of work in fixed point theory in various spaces with graph has been done by changing some conditions on mapping and spaces, by redefining some definitions on mappings like G-contraction, G-kannan, G-monotone non-expansive, $\varphi$ -contraction, G- graphic contraction, and etc.

Now, we recall some basic definitions, notations and relevant concepts to partial metric space and common fixed point theorem.

Let $\mathrm{M}$ be any non-empty set. A partial metric $\mathrm{p}$ on $\mathrm{M}$ differ from ordinary metric $\mathrm{d}$ on $\mathrm{M}$ with two following property:

1) $\quad p(x, \varkappa)=p(y, y)=p(x, y)$ iff $x=y$

i.e, $\mathrm{p}(\mathcal{x}, y)=0 \Rightarrow \varkappa=y$ but if $\varkappa=y$ doesn't imply $\mathrm{p}(\varkappa, y)=0$. Also, p $(\varkappa, \varkappa) \leq p(\varkappa, y)$.

2) $\mathrm{p}(\mathcal{H}, y) \leq \mathrm{p}(\mathcal{X}, z)+\mathrm{p}(z, y)-\mathrm{p}(z, z)$ for all $\mathcal{y}, y, z \in$ M.

Let $\mathrm{M}$ be partial metric space with partial metric $p$, let sequence $\left\{\varkappa_{\mathrm{n}}\right\}$ in $\mathrm{M}$ and $\mathcal{\varkappa} \in \mathrm{M}$. Then we have

1) $\left\{\varkappa_{\mathrm{n}}\right\}$ is convergent to $\mathcal{x}$ if $\lim _{n \rightarrow \infty} \mathrm{p}\left(\mathcal{x}, \varkappa_{\mathrm{n}}\right)=\mathrm{p}(\mathcal{x}, \mathcal{x})$;

2) $\left\{\varkappa_{n}\right\}$ is called a Cauchy sequence if there exists $\lim _{n \rightarrow \infty} \mathrm{p}\left(\mathcal{\varkappa}_{\mathrm{n}}, \mathcal{H}_{\mathrm{m}}\right)$ such that it is finite;

3) MiscompleteifeveryCauchysequenceinMisconvergen ttoapoint $\mathcal{H} \in$ Msuchthat $\lim _{n, m \rightarrow \infty} \mathrm{p}\left(\mathcal{H}_{\mathrm{n},}, \mathcal{H}_{\mathrm{m}}\right)=\mathrm{p}(\mathcal{\varkappa}, \mathcal{\varkappa})$

4) Ifthesequence $\left\{\mathcal{H}_{\mathrm{n}}\right\}$ convergesto $\mathcal{K} \in \operatorname{Mandp}(\mathcal{H}, \mathcal{K})=0$, th en $\lim _{n \rightarrow \infty} \mathrm{p}\left(\varkappa_{\mathrm{n}}, y\right)=\mathrm{p}(\mathcal{x}, y)$, foreveryy $\in \mathrm{M}$

Definition 1.2. [4] Suppose that $f$ and $g_{s}$ are two mappings from a metric space $(\mathrm{M}, \mathrm{d})$ into itself. Then

i) The mapping are said to be commutating if $\mathrm{fg}_{\mathrm{g}} \mathcal{H}$ $=g_{5} f x, \forall \varkappa \in M$.

ii) The mapping is compatible if $\mathrm{d}\left(\mathrm{fg}_{\mathcal{O}} \mathcal{\varkappa}_{\mathrm{n}}, \mathrm{g} f \mathrm{f} \mathcal{\varkappa}_{\mathrm{n}}\right) \rightarrow 0$ as $\mathrm{n} \rightarrow \infty$ whenever $\left\{\mathcal{H}_{\mathrm{n}}\right\}$ is a sequence in $\mathrm{M}$ such that $\mathrm{f} \mathcal{H}_{\mathrm{n}}, \mathrm{g}_{\mathcal{O}} \mathcal{H}_{\mathrm{n}} \rightarrow \mathrm{t}$, for some $\mathrm{t} \epsilon \mathrm{M}$ and weakly compatible if they commute at their coincidence point i.e, if $\mathrm{f} \mathcal{X}=$

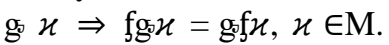

iii) The mapping $f$ and $g_{s}$ are said to be semi-compatible mapping if $\left\{\varkappa_{\mathrm{n}}\right\}$ be a sequence in $\mathrm{M}$ such thatf $\mathcal{\varkappa}_{\mathrm{n}} \rightarrow \mathcal{\varkappa}$, $\mathrm{g}_{\mathrm{o}} \mathrm{X}_{\mathrm{n}} \rightarrow \mathcal{H}$ implies $\mathrm{f}_{0} \boldsymbol{\varkappa}_{\mathrm{n}} \rightarrow \mathrm{g}_{3} \mathcal{\varkappa}$ and both mappings commute at their coincidence point and semi-weakly compatible if they commute at the fixed point of either $f$ or $g_{0}$

i.e, If $\mathrm{f}_{3}=3$ or $\mathrm{g}_{3} 3=3$ then $\mathrm{fg}_{3} 3=g_{3} f_{3}$.

Remark: Weakly compatible and semi-weakly compatible are independent of each other.

Proposition 1.3. For two self-maps on metric space (M, d), the notion of commutativity $\Rightarrow$ weakly commutativity $\Rightarrow$ compatibility $\Rightarrow$ weakly compatibility $\Rightarrow$ commutativity at common fixed points, but the convers are not true always.

Definition 1.4. [9] A mapping f: $M \rightarrow M$ is a Banach $\mathrm{G}$-contraction if $\mathrm{f}$ preserves edges of $\mathrm{G}$ i.e., $\forall \mathcal{H}, y \in \mathrm{M}$ $((\varkappa, y) \in \mathrm{E}(\mathrm{G}) \Rightarrow(\mathrm{f} \mathcal{\mathrm { f }}, \mathrm{f} \mathrm{y}) \in \mathrm{E}(\mathrm{G}))$

and $f$ decreases weights of edges of $G$ in the following way:

$$
\begin{gathered}
\exists \alpha \in(0,1) \forall \varkappa, y \in \mathrm{X}((\mathcal{H}, \mathrm{y}) \in \mathrm{E}(\mathrm{G}) \\
\Rightarrow \mathrm{d}(\mathrm{f} \mathcal{H}, \mathrm{f} y) \leq \alpha \mathrm{d}(\mathcal{H}, \mathrm{y})) .
\end{gathered}
$$

Definition 1.5. [9] A mapping $\mathrm{f}: \mathrm{M} \rightarrow \mathrm{M}$ is called G-continuous if given $\varkappa \in \mathrm{M}$ and a sequence $\left(\varkappa_{n}\right)_{n \in N}$, $\varkappa_{n} \rightarrow \mathcal{H}$ and $\left(\mathcal{H}_{n}, \mathcal{\varkappa}_{n+1}\right) \in \mathrm{E}(\mathrm{G})$ for $\mathrm{n} \in \mathrm{N}$ impliesf $\mathcal{H}_{n} \rightarrow$

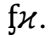

Theorem 1.6. [11] If $p$ is a partial metric, $M$ be a non-empty set then the function $d_{p}: M \times M \rightarrow[0, \infty)$ given by

$$
\mathrm{d}_{\mathrm{p}}(\varkappa, y)=2 p(\varkappa, y)-\frac{p(\varkappa, \varkappa)-p(y, y) \text { for all } \varkappa, y \in}{M}
$$

is a metric on $\mathrm{M}$, called the associated metric to $\mathrm{p}$.

Lemma 1.7. [11] Let $(M, p)$ be a partial metric space then $\left\{\mathcal{H}_{\mathrm{n}}\right\}$ is a Cauchy sequence in $(\mathrm{M}, \mathrm{p})$ if and only if it is a Cauchy sequence in the metric space $\left(M, d_{p}\right)$.

Lemma 1.8. Let $\left\{\varkappa_{n}\right\}$ and $\left\{y_{n}\right\}$ be two sequences in partial metric space $(\mathrm{M}, \mathrm{p})$ such that

$$
\lim _{n \rightarrow \infty} p\left(\varkappa_{n}, y_{n}\right)=0 \text {. }
$$

If $\left\{\mathcal{H}_{n}\right\}$ is Cauchy sequence, so $\left\{y_{n}\right\}$ is.

Proof: Let $m>n$.

First, we compute

$$
\begin{array}{r}
\mathrm{p}\left(\mathrm{y}_{n}, \mathrm{y}_{n}\right) \leq \mathrm{p}\left(\mathrm{y}_{n}, \varkappa_{n}\right)+\mathrm{p}\left(\varkappa_{n}, y_{n}\right)-\mathrm{p}\left(\varkappa_{n}, x_{n}\right) \leq \\
\mathrm{p}\left(\mathrm{y}_{n}, \varkappa_{n}\right)+\mathrm{p}\left(\varkappa_{n}, y_{n}\right)=2 \mathrm{p}\left(\varkappa_{n}, y_{n}\right) .
\end{array}
$$

Taking limit $\mathrm{n} \rightarrow \infty$, we get

$$
0 \leq \lim _{n \rightarrow \infty} \mathrm{p}\left(y_{n}, y_{n}\right) \leq 2 \lim _{n \rightarrow \infty} \mathrm{p}\left(\mathcal{H}_{n}, y_{n}\right)=0
$$

i.e.,

$$
\lim _{n \rightarrow \infty} \mathrm{p}\left(\mathrm{y}_{n}, y_{n}\right)=0
$$

From the Theorem 1.6., corresponding to every partial metric $p$, we can define the metric

$$
\mathrm{d}_{\mathrm{p}}(\mathcal{H}, y)=2 \mathrm{p}(\mathcal{x}, y)-\mathrm{p}(\mathcal{x}, \mathcal{x})-\mathrm{p}(y, y) .
$$

For $\varkappa=\varkappa_{\mathrm{n}}$ and $y=y_{n}$, it becomes

$$
\mathrm{d}_{\mathrm{p}}\left(\varkappa_{\mathrm{n}}, y_{\mathrm{n}}\right)=2 \mathrm{p}\left(\varkappa_{\mathrm{n}}, y_{\mathrm{n}}\right)-\mathrm{p}\left(\varkappa_{n}, \varkappa_{n}\right)+\mathrm{p}\left(y_{n}, y_{n}\right) .
$$


Taking limit $\mathrm{n} \rightarrow \infty$ and by (1), (2), we get

$$
\begin{aligned}
\lim _{n \rightarrow \infty} d_{\mathrm{p}}\left(\mathcal{H}_{\mathrm{n}}, y_{\mathrm{n}}\right)=2 \lim _{n \rightarrow \infty} \mathrm{p}\left(\mathcal{\varkappa}_{\mathrm{n}}, y_{\mathrm{n}}\right)-\lim _{n \rightarrow \infty}\left[\mathrm{p}\left(\mathcal{\varkappa}_{n}, \mathcal{\varkappa}_{n}\right)+\right. \\
\left.\mathrm{p}\left(y_{n}, y_{n}\right)\right] \\
\lim _{n \rightarrow \infty} d_{p}\left(\mathcal{X}_{\mathrm{n}}, y_{\mathrm{n}}\right)=0 .
\end{aligned}
$$

Next,

$$
\mathrm{d}_{\mathrm{p}}\left(\mathrm{y}_{\mathrm{m}}, y_{\mathrm{n}}\right) \leq \mathrm{d}_{\mathrm{p}}\left(\mathrm{y}_{\mathrm{m}}, \varkappa_{m}\right)+\mathrm{d}_{\mathrm{p}}\left(\varkappa_{m}, \varkappa_{n}\right)+\mathrm{d}_{\mathrm{p}}\left(\varkappa_{\mathrm{n}}, y_{\mathrm{n}}\right)
$$

Applying limit $\mathrm{n}, \mathrm{m} \rightarrow \infty$ and by Lemma 1.7, (3), it follows that

$$
\begin{gathered}
\lim _{n, m \rightarrow \infty} \mathrm{d}_{\mathrm{p}}\left(\mathrm{Y}_{\mathrm{m}}, \mathrm{Y}_{\mathrm{n}}\right) \leq \lim _{m \rightarrow \infty} \mathrm{d}_{\mathrm{p}}\left(\mathrm{Y}_{\mathrm{m}}, \mathcal{\varkappa}_{m}\right)+\lim _{n, m \rightarrow \infty} \mathrm{d}_{\mathrm{p}}\left(\mathcal{\varkappa}_{\mathrm{m}}, \mathcal{\varkappa}_{n}\right) \\
+\lim _{n \rightarrow \infty} \mathrm{d}_{\mathrm{p}}\left(\mathcal{U}_{\mathrm{n}}, \mathrm{Y}_{\mathrm{n}}\right) \\
=2 \lim _{n \rightarrow \infty} \mathrm{d}_{\mathrm{p}}\left(\mathcal{\varkappa}_{\mathrm{n}}, \mathrm{Y}_{\mathrm{n}}\right)+\lim _{n, m \rightarrow \infty} \mathrm{d}_{\mathrm{p}}\left(\mathcal{\varkappa}_{\mathrm{m}}, \mathcal{\varkappa}_{\mathrm{n}}\right)
\end{gathered}
$$

$=0$

which implies $\left\{y_{n}\right\}$ is Cauchy sequence in metric space $\left(\mathrm{M}, \mathrm{d}_{\mathrm{p}}\right)$.

Therefore by Lemma 1.7., it is also Cauchy sequence in partial metric space (M, p).

Throughout this paper, suppose triplet $(\mathrm{M}, \mathrm{p}, \mathrm{G})$ be a partial metric space, $G$ be a directed graph where the set $V(G)=M$ and the edge set $E(G)$ contains no parallel edges such that $\Delta \subseteq \mathrm{E}(\mathrm{G}), \Delta$ denote the diagonals of the Cartesian product $\mathrm{M} \times \mathrm{M}$. $\tilde{G}$ means the undirected graph which is obtained from $\mathrm{G}$ by ignoring the direction of edges and $\mathrm{E}(\tilde{G})=\mathrm{E}(\mathrm{G}) \cup \mathrm{E}\left(G^{-1}\right)$.

Inspired by the some recent work on metric spaces with a graph (see [1,2,7-9,15]), we establish some interesting common fixed point results from metric space to partial metric space through a new approach. In this paper, we obtain some common fixed point theorems in a complete partial metric space endowed with graph using $\varepsilon$-compatible maps.

\section{Main Results}

In this section, we present some new type of common fixed point theorems by using the concept of $\varepsilon$-compatible in context of partial metric space and also obtain some examples to understand the usability of the definitions and results.

Definition 2.1. [11]. Two self-maps $f$ and $g$ on a metric space $(\mathrm{M}, \mathrm{d})$ are $\boldsymbol{\varepsilon}$-compatible if for every $\varepsilon>0$, there exists an element $\mathcal{K} \in \mathrm{M}$ such that $\mathrm{d}(\mathrm{f} \mathcal{H}, \mathrm{g} \mathcal{X})<\varepsilon$ implies $\mathrm{d}(\mathrm{fg} \mathcal{\mathcal { H }}$, gf $\mathcal{X})<\varphi(\varepsilon)$, where $\varphi:[0, \infty) \rightarrow[0, \infty)$ is continuous at 0 .

Remark: If there exists atleast one coincidence point and the mapping $\mathrm{f}, \mathrm{g}$ commute at that coincidence point then they are $\varepsilon$-compatible but converse needs not to be true. For example: Let $\mathrm{M}=\mathrm{R}$ be a metric space with a usual metric $\mathrm{d}(\varkappa, y)=|\varkappa-y|$. Two self -mapping $\mathrm{f}$ and $\mathrm{g}$ on Mare defined as $\mathrm{f}(\mathcal{X})=e^{-\varkappa}+1$ and $\mathrm{g}(\mathcal{K})=1$ are $\varepsilon$ -compatible but they have no coincidence point.

Example 2.2. Let $M=R$ be a metric space with a usual metric $\mathrm{d}(\mathcal{X}, y)=|\mathcal{U}-y|$. Define $\mathrm{f}, \mathrm{g}: \mathrm{M} \rightarrow \mathrm{M}$ by

$$
\mathrm{f} \varkappa=\left\{\begin{array}{c}
\varkappa^{2}, \varkappa \neq 1 \\
3, \varkappa=1
\end{array} \text { and } \mathrm{g} \varkappa=3 \varkappa \text { for all } \varkappa \in \mathrm{M}\right. \text {. }
$$

For any $\varepsilon>0$, whatever we take there always exists $\mathcal{X}=0$, $\mathcal{\varkappa}=1$ such that $|\mathrm{f} \mathcal{H}-\mathrm{g} \mathcal{X}|=0$ and $|\mathrm{fg} \mathcal{X}-\mathrm{gf} \mathcal{H}|=0$, for any function $\varphi$ where $\varphi:[0, \infty) \rightarrow[0, \infty)$ is continuous function at 0 . That is, the pair $\mathrm{f}$ and $\mathrm{g}$ is $\varepsilon$-compatible. For $\mathcal{\varkappa}=3, \mathrm{f} \mathcal{\varkappa}=\mathrm{g} \mathcal{X}=9$, but $\mid \mathrm{fg} \mathcal{H}$ - gf $\mathcal{H} \mid \neq 0$. Hence, the pair $\mathrm{f}$ and $g$ are not compatible and also not weakly compatible.

Example 2.3. From the Figure 1., we can easily observe that any two parallel graphs on $\mathrm{R}$ never are $\varepsilon$-compatible since they have the fixed distance so that they never come to close at any point therefore, they are not $\varepsilon$-compatible but they are always compatible since these graph are translation maps.

For an example, if we take $\mathrm{f} \varkappa=\varkappa+3$ and $\mathrm{g} \varkappa=\varkappa+7$ then $\mathrm{f}$ and $\mathrm{g}$ are compatible, infact commutating maps are not $\varepsilon$-compatible because $|\mathrm{f} \varkappa-\mathrm{g} \varkappa|=4$ for all $\varkappa \in \mathrm{M}$ and if we take any $\varepsilon<4$, we couldn't find an element $\varkappa \in \mathrm{M}$ such that $\mathrm{d}(\mathrm{f} \mathcal{X}, \mathrm{g} \mathcal{X})<\varepsilon$ i.e, this is the necessary condition for $\varepsilon$-compatible mappings.

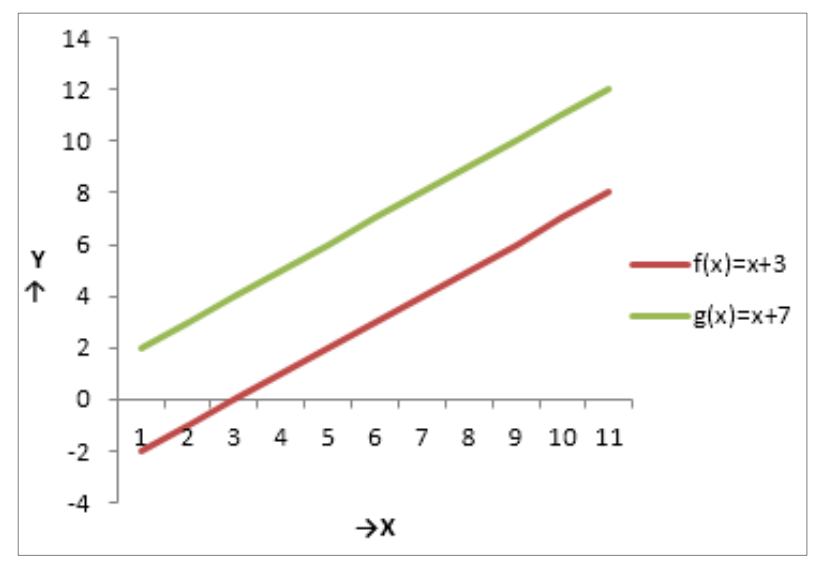

Figure 1. Maps $\mathrm{f}$ and $\mathrm{g}$ with fixed distance are not $\varepsilon$-compatible

Remark: The concept of $\varepsilon$-compatible is independent of weakly compatible and compatible.

Theorem 2.4. Let the triplet $(\mathrm{M}, \mathrm{p}, \mathrm{G})$ be a complete partial metric space endowed with a graph $\mathrm{G}$ and $\mathrm{f}, \mathrm{g}$ be $\varepsilon$ -compatible mappings on $\mathrm{M}$ which satisfies the condition

$\mathrm{p}(\mathrm{g} \mathcal{U}, \mathrm{gy}) \leq \mathrm{a} p(\mathrm{f} \mathcal{H}, \mathrm{f} y)+\mathrm{b} p(\mathrm{f} \mathcal{H}, \mathrm{gy})+\mathrm{c} p(\mathrm{f} y, \mathrm{~g} \mathcal{X})$,

$$
\forall(\mathcal{H}, y) \in \mathrm{E}(\tilde{G})(4)
$$

where $a, b, c$ are in $R_{+}$such that $a+b+c \neq 1, a+b+c<1$.

Assume that $f$ is $G$ - continuous with the following property:

(*)As $\mathrm{f}$ and $\mathrm{g}$ are $\varepsilon$ - compatible pair, so for every $\varepsilon(n)>0$, there exists an element $\mathcal{\varkappa}_{\mathrm{n}}$ in M such thatp $\left(\mathrm{f} \mathcal{\varkappa}_{n}\right.$, $\left.\mathrm{g} \mathcal{\varkappa}_{n}\right)<\varepsilon(n) \Rightarrow \mathrm{p}\left(\mathrm{fg} \mathcal{\varkappa}_{n}, \mathrm{gf} \mathcal{\varkappa}_{n}\right)<\varphi(\varepsilon(n))$ then $\left(\varkappa_{n}, \varkappa_{n+1}\right)$, $\left(\mathrm{f} \varkappa_{n}, \mathrm{f} \mathcal{H}_{n+1}\right) \in \mathrm{E}(\tilde{G})$ and $\left(\mathrm{g} \mathcal{H}_{n}, \mathrm{~g} \mathcal{H}_{n+1}\right) \in \mathrm{E}(\tilde{G})(\varepsilon(n)$ depends on $\mathrm{n}$ and take value less than one );

$(* *)$ If a sequence $\mathrm{f} \varkappa_{n} \rightarrow \varkappa$ then $\left(\mathrm{f} \varkappa_{n}, \mathcal{\varkappa}\right) \in \mathrm{E}(\tilde{G})$ and $\left(\varkappa_{n}\right.$, $\varkappa) \epsilon \mathrm{E}(\tilde{G})$. Further $\mathrm{f}$ satisfies the condition $\operatorname{ifp}(\mathcal{\varkappa}, \mathcal{\varkappa})=0$ then $\mathrm{p}(\mathrm{f} \mathcal{U}, \mathrm{f} \mathcal{H})=0$. 
Then $\mathrm{f}$ and $\mathrm{g}$ have common fixed point. Furthermore, if $\mathcal{H}, \mathcal{H}^{*}$ are fixed points of $\mathrm{f}$ and $\mathrm{g}$ with $\left(\mathcal{H}, \mathcal{\varkappa}^{*}\right) \in \mathrm{E}(\tilde{G})$ then $\mathrm{f}$ and $\mathrm{g}$ have unique common fixed point.

Proof: Since $f$ and $g$ are $\varepsilon$-compatible, so by their $\varepsilon$-compatibility for every $\mathrm{n} \epsilon \mathrm{N}$ and for every $\varepsilon>0$, take $\varepsilon$ $=1 / \mathrm{n}^{2}$ there always exists a sequence $\left\{\mathcal{H}_{\mathrm{n}}\right\}$ in $\mathrm{M}$ such that

$$
\mathrm{p}\left(\mathrm{f} \mathcal{\varkappa}_{\mathrm{n}}, \mathrm{g} \mathcal{\varkappa}_{\mathrm{n}}\right)<\frac{1}{n^{2}} \Rightarrow \mathrm{p}\left(\mathrm{fg} \mathcal{\varkappa}_{\mathrm{n}}, \mathrm{gf} \mathcal{\varkappa}_{\mathrm{n}}\right)<\varphi\left(\frac{1}{n^{2}}\right) .
$$

Now, we have by using (4) and $(*)$

$\mathrm{p}\left(\mathrm{g} \mathcal{\varkappa}_{\mathrm{n}}, \mathrm{g} \mathcal{\varkappa}_{\mathrm{n}+1}\right) \leq \mathrm{ap}\left(\mathrm{f} \mathcal{\varkappa}_{\mathrm{n}}, \mathrm{f} \mathcal{\varkappa}_{\mathrm{n}+1}\right)+\mathrm{b} \mathrm{p}\left(\mathrm{f} \mathcal{\varkappa}_{\mathrm{n}}, \mathrm{g} \mathcal{X}_{\mathrm{n}+1}\right)+\mathrm{c}$ $\mathrm{p}\left(\mathrm{f} \mathcal{\varkappa}_{\mathrm{n}+1}, \mathrm{~g} \mathcal{\varkappa}_{\mathrm{n}}\right) \leq \mathrm{a}\left[\mathrm{p}\left(\mathrm{f} \mathcal{\varkappa}_{\mathrm{n}}, \mathrm{g} \mathcal{\varkappa}_{\mathrm{n}}\right)+\mathrm{p}\left(\mathcal{\varkappa}_{\mathrm{n}}, \mathrm{g} \mathcal{\varkappa}_{\mathrm{n}+1}\right)+\mathrm{p}\left(\mathrm{g} \mathcal{\varkappa}_{\mathrm{n}+1}\right.\right.$, $\left.\left.\mathrm{f} \mathcal{\varkappa}_{\mathrm{n}+1}\right)\right]+\mathrm{b}\left[\mathrm{p}\left(\mathrm{f} \mathcal{\varkappa}_{\mathrm{n}}, \mathrm{gx} \mathcal{\varkappa}_{\mathrm{n}}\right)+\mathrm{p}\left(\mathrm{g} \mathcal{\varkappa}_{\mathrm{n}}, \mathrm{g} \mathcal{\varkappa}_{\mathrm{n}+1}\right)\right]+\mathrm{c}\left[\mathrm{p}\left(\mathrm{f} \mathcal{\varkappa}_{\mathrm{n}+1}, \mathrm{~g} \mathcal{\varkappa}_{\mathrm{n}+1}\right)\right.$ $\left.+\mathrm{p}\left(\mathrm{g} \mathcal{\varkappa}_{\mathrm{n}+1}, \mathrm{~g} \mathcal{\varkappa}_{\mathrm{n}}\right)\right]$

implies that

$$
\begin{aligned}
& (1-(\mathrm{a}+\mathrm{b}+\mathrm{c})) \mathrm{p}\left(\mathrm{g} \mathcal{\varkappa}_{\mathrm{n}}, \mathrm{g} \mathcal{\varkappa}_{\mathrm{n}+1}\right) \\
& \quad \leq(\mathrm{a}+\mathrm{b}) \mathrm{p}\left(\mathrm{f} \mathcal{\varkappa}_{\mathrm{n}}, \mathrm{g} \mathcal{\varkappa}_{\mathrm{n}}\right)+(\mathrm{a}+\mathrm{c}) \mathrm{p}\left(\mathrm{f} \mathcal{\varkappa}_{\mathrm{n}+1}, \mathrm{~g} \mathcal{\varkappa}_{\mathrm{n}+1}\right) \\
& \Rightarrow \quad \mathrm{p}\left(\mathrm{g} \mathcal{\varkappa}_{\mathrm{n}}, \mathrm{g} \mathcal{\varkappa}_{\mathrm{n}+1}\right) \\
& \leq \frac{\mathrm{a}+\mathrm{b}}{1-(\mathrm{a}+\mathrm{b}+\mathrm{c})} \mathrm{p}\left(\mathrm{f} \mathcal{\varkappa}_{\mathrm{n}}, \mathrm{g} \mathcal{\varkappa}_{\mathrm{n}}\right)+\frac{\mathrm{a}+\mathrm{c}}{1-(\mathrm{a}+\mathrm{b}+\mathrm{c})} \mathrm{p}\left(\mathrm{f} \mathcal{\varkappa}_{\mathrm{n}+1}, \mathrm{~g} \mathcal{U}_{\mathrm{n}+1}\right) \\
& \quad<\frac{\mathrm{a}+\mathrm{b}}{1-(\mathrm{a}+\mathrm{b}+\mathrm{c})} \frac{1}{n^{2}}+\frac{\mathrm{a}+\mathrm{c}}{1-(\mathrm{a}+\mathrm{b}+\mathrm{c})} \frac{1}{(n+1)^{2}} \\
& <\frac{2 \mathrm{a}+\mathrm{b}+\mathrm{c}}{1-(\mathrm{a}+\mathrm{b}+\mathrm{c})} \frac{1}{n^{2}} .
\end{aligned}
$$

For $\mathrm{m} \leq n$, we have

$\mathrm{p}\left(\mathrm{g} \mathcal{\varkappa}_{\mathrm{m}}, \mathrm{g} \mathcal{\varkappa}_{\mathrm{n}}\right) \leq \sum_{k=m}^{n-1} \mathrm{p}\left(\mathrm{g} \mathcal{\varkappa}_{k}, \mathrm{~g} \varkappa_{k+1}\right) \leq \frac{2 \mathrm{a}+\mathrm{b}+\mathrm{c}}{1-(\mathrm{a}+\mathrm{b}+\mathrm{c})} \sum_{m}^{n-1} \frac{1}{k^{2}}$.

Since the series $\sum \frac{1}{n^{2}}$ converges, therefore the sequence $\left\{\mathrm{g} \mathcal{H}_{\mathrm{n}}\right\}$ is Cauchy and lemma $\left\{\mathrm{f} \mathcal{H}_{\mathrm{n}}\right\}$ is also Cauchy. As $\mathrm{M}$ is complete, there exists u $\epsilon \mathrm{M}$ such that the sequences $\left\{\mathrm{g} \mathcal{H}_{\mathrm{n}}\right\}$ and $\left\{\mathrm{f} \mathcal{\varkappa}_{\mathrm{n}}\right\}$ converges to $\mathrm{u}$, since $\mathrm{p}\left(\mathrm{f} \mathcal{H}_{\mathrm{n}}, \mathrm{g} \mathcal{\varkappa}_{\mathrm{n}}\right) \rightarrow 0$ as $\mathrm{n} \rightarrow \infty$ and

$\lim _{n \rightarrow \infty} \mathrm{p}\left(\mathrm{g} \mathcal{U}_{\mathrm{n}}, \mathrm{u}\right)=\lim _{n, m \rightarrow \infty} \mathrm{p}\left(\mathrm{g} \mathcal{H}_{\mathrm{n}}, \mathrm{g} \mathcal{H}_{\mathrm{m}}\right)=\mathrm{p}(\mathrm{u}, \mathrm{u})=\lim _{n, m \rightarrow \infty} \mathrm{p}\left(\mathrm{f} \mathcal{H}_{\mathrm{n}}\right.$, $\left.\mathrm{f} \mathcal{H}_{\mathrm{m}}\right)=\lim _{n \rightarrow \infty} \mathrm{p}\left(\mathrm{f} \mathcal{\varkappa}_{\mathrm{n}}, \mathrm{u}\right)$

But $\lim _{n \rightarrow \infty} \mathrm{p}\left(\mathrm{g} \mathcal{\varkappa}_{\mathrm{n}}, \mathrm{g} \mathcal{\varkappa}_{\mathrm{m}}\right)=0$, therefore $\mathrm{p}(\mathrm{u}, \mathrm{u})=0$ and by $(* *)$, $p(f u, f u)=0$.

G-continuity of $\mathrm{f}$ and by the condition $(*)$,

fg $\mathcal{H}_{\mathrm{n}}, \mathrm{f}^{2} \mathcal{H}_{\mathrm{n}} \rightarrow$ fu and $\mathrm{p}\left(\mathrm{fg} \mathcal{H}_{\mathrm{n}}, \mathrm{gf} \mathcal{H}_{\mathrm{n}}\right) \rightarrow 0$ as $\mathrm{n} \rightarrow \infty$ with $\mathrm{p}(\mathrm{fu}, \mathrm{fu})=0$ implies $\left\{\mathrm{g} f \mathcal{H}_{\mathrm{n}}\right\}$ converges to fu. Suppose that $\mathrm{fu} \neq \mathrm{gu}$ then $\mathrm{p}(\mathrm{fu}, \mathrm{gu})>0$.

Now, we have using (4) and $(* *)$

$\mathrm{p}\left(\mathrm{gf} \mathcal{\varkappa}_{\mathrm{n}}, \mathrm{gu}\right) \leq \mathrm{a} p\left(\mathrm{ff} \mathcal{\varkappa}_{\mathrm{n}}, \mathrm{fu}\right)+\mathrm{b} \mathrm{p}\left(\mathrm{ff} \mathcal{\varkappa}_{\mathrm{n}}, \mathrm{gu}\right)+\mathrm{c} p\left(\mathrm{fu}, \mathrm{gf} \mathcal{\varkappa}_{\mathrm{n}}\right)$.

Taking $\mathrm{n} \rightarrow \infty$, we obtain

$p(f u, g u) \leq a p(f u, f u)+b p(f u, g u)+c p(f u, f u)$

$=(\mathrm{a}+\mathrm{c}) \mathrm{p}(\mathrm{fu}, \mathrm{fu})+\mathrm{b} p(f u, g u)$

$\leq \mathrm{b} p(\mathrm{fu}, \mathrm{gu})($ since, $\mathrm{p}(\mathcal{\varkappa}, \mathcal{U}) \leq \mathrm{p}(\mathcal{H}, \mathrm{y}) \forall \varkappa, y \in M)$

$\Rightarrow(1-b) \quad p(f u, g u)<0$

$\Rightarrow p(\mathrm{fu}, \mathrm{gu})=0$

which is a contradiction, thus we have shown that fu=gu.
Again, Using (4) and (**)

$\mathrm{p}\left(\mathrm{g} \mathcal{H}_{\mathrm{n}}, \mathrm{gu}\right) \leq \mathrm{a} p\left(\mathrm{f} \mathcal{H}_{\mathrm{n}}, \mathrm{fu}\right)+\mathrm{b} \mathrm{p}\left(\mathrm{f} \mathcal{\varkappa}_{\mathrm{n}}, \mathrm{gu}\right)+\mathrm{c} \mathrm{p}\left(\mathrm{fu}, \mathrm{g} \mathcal{\varkappa}_{\mathrm{n}}\right)$

Letting $\mathrm{n} \rightarrow \infty$,

$p(u, g u) \leq a p(u, f u)+b p(u, g u)+c p(f u, u)$

$=(a+b+c) p(u, g u)$

i.e., $(1-(a+b+c)) p(u, g u)<0$ implies $p(u, g u)=0 \Rightarrow g u=u$

So, we get

$$
\mathrm{fu}=\mathrm{gu}=\mathrm{u} \text {. }
$$

Now, we show that $\mathrm{u}$ is the unique fixed point. Let $\mathrm{v} \neq$ $\mathrm{u}$ be a point in $\mathrm{M}$ such that $\mathrm{fv}=\mathrm{gv}=\mathrm{v}$, then by (2), we get

$p(g u, g v) \leq a p(f u, f v)+b p(f u, g v)+c p(f v, g u)$

$$
p(u, v) \leq a p(u, v)+b p(u, v)+c p(v, u)
$$

i.e., $(1-(a+b+c)) p(u, v) \leq 0$

implies that $\mathrm{p}(\mathrm{u}, \mathrm{v})=0$ i.e, $\mathrm{u}=\mathrm{v}$.

Hence $f$ and $g$ have a unique common fixed point.

Corollary 2.5. Letf and $g$ be the self-mapping of a complete partial metric space $(\mathrm{M}, \mathrm{p})$ endowed with a graph $\mathrm{G}=(\mathrm{V}, \mathrm{E})$ which satisfies the condition

$$
\mathrm{p}(\mathrm{g} \mathcal{\mathrm { g }} \mathrm{g} \mathrm{y}) \leq \alpha \mathrm{p}(\mathrm{f} \mathcal{f}, \mathrm{fy}), \forall(\mathrm{f} \mathcal{f}, \mathrm{fy}) \epsilon \mathrm{E}(\tilde{G})
$$

where $\alpha \epsilon(0,1)$. Assume that $\mathrm{f}$ and $\mathrm{g}$ are $\varepsilon$-compatible pair with the following property:

(*) As $\mathrm{f}$ and $\mathrm{g}$ are $\varepsilon$ - compatible pair, so for every $\varepsilon(n)>0$, there exists an element $\varkappa_{\mathrm{n}}$ in M such that $\mathrm{p}\left(\mathrm{f} \mathcal{H}_{\mathrm{n}}\right.$, $\left.\mathrm{g} \mathcal{H}_{\mathrm{n}}\right)<\varepsilon(n) \Rightarrow \mathrm{p}\left(\mathrm{fg} \mathcal{H}_{\mathrm{n}}, \mathrm{gf} \mathcal{H}_{\mathrm{n}}\right)<\varphi(\varepsilon(n))$ then $\left(\mathrm{f} \mathcal{H}_{\mathrm{n}}, \mathrm{f} \mathcal{H}_{\mathrm{n}+1}\right)$ $\epsilon \mathrm{E}(\tilde{G})$ and $\left(\mathrm{g} \mathcal{H}_{\mathrm{n}}, \mathrm{g} \mathcal{H}_{\mathrm{n}+1}\right) \epsilon \mathrm{E}(\widetilde{G})(\varepsilon(n)$ depends on $\mathrm{n}$ or can say function of $\mathrm{n}$ );

(**) Suppose $\mathrm{f}$ be a $\mathrm{G}$ - continuous map i.e., if a sequence $\left\{\varkappa_{n}\right\} \rightarrow \mathcal{K}$ and $\left(\varkappa_{n}, \varkappa_{n+1}\right) \in \mathrm{E}(\tilde{G})$ thenf $\mathcal{H}_{n} \rightarrow \mathrm{f} \mathcal{K}$ with condition $\left(\mathrm{f} \mathcal{H}_{n}, \mathrm{f} \mathcal{U}\right) \in \mathrm{E}(\tilde{G})$ and $\left(\mathcal{H}_{n}, \mathrm{f} \mathcal{X}\right) \in \mathrm{E}(\tilde{G})$. Further $\mathrm{f}$ satisfies the condition if $\mathrm{p}(\mathcal{\varkappa}, \mathcal{\varkappa})=0$ then $\mathrm{p}(\mathrm{f} \mathcal{H}$, $\mathrm{f} \mathcal{X})=0$.

Then $\mathrm{f}$ and $\mathrm{g}$ have unique common fixed point.

Proof: Since $\mathrm{f}$ and $\mathrm{g}$ are $\varepsilon$ - compatible pair, so for every $\varepsilon>0$, take $\varepsilon=1 / \mathrm{n}^{2}$, there exists an element $\mathcal{H}_{\mathrm{n}}$ in M such that $\mathrm{p}\left(\mathrm{f} \mathcal{H}_{\mathrm{n}}, \mathrm{g} \mathcal{H}_{\mathrm{n}}\right)<\frac{1}{n^{2}} \Rightarrow \mathrm{p}\left(\mathrm{fg} \mathcal{H}_{\mathrm{n}}, \mathrm{gf} \mathcal{H}_{\mathrm{n}}\right)<\varphi\left(\frac{1}{n^{2}}\right)$.

Now for $\mathcal{X}=\mathcal{\varkappa}_{\mathrm{n}}, y=\mathcal{\varkappa}_{\mathrm{n}+1}$, and using $(*)$ we have

$$
\mathrm{p}\left(\mathrm{g} \mathcal{H}_{\mathrm{n}}, \mathrm{g} \mathcal{\varkappa}_{\mathrm{n}+1}\right) \leq \alpha \mathrm{p}\left(\mathrm{f} \mathcal{\varkappa}_{\mathrm{n}}, \mathrm{f} \mathcal{\varkappa}_{\mathrm{n}+1}\right) .
$$

By the triangle inequality

$$
\begin{gathered}
\mathrm{p}\left(\mathrm{f} \mathcal{\varkappa}_{\mathrm{n}}, \mathrm{f} \mathcal{\varkappa}_{\mathrm{n}+1}\right) \leq \mathrm{p}\left(\mathrm{f} \mathcal{\varkappa}_{\mathrm{n}}, \mathrm{g} \mathcal{\varkappa}_{\mathrm{n}}\right)+\mathrm{p}\left(\mathrm{g} \mathcal{\varkappa}_{\mathrm{n}}, \mathrm{f} \mathcal{\varkappa}_{\mathrm{n}+1}\right)-\mathrm{p}\left(\mathrm{g} \mathcal{\varkappa}_{\mathrm{n}}, \mathrm{g} \mathcal{\varkappa}_{\mathrm{n}}\right) \\
<\mathrm{p}\left(\mathrm{f} \mathcal{\varkappa}_{\mathrm{n}}, \mathrm{g} \mathcal{\varkappa}_{\mathrm{n}}\right)+\mathrm{p}\left(\mathrm{g} \mathcal{\varkappa}_{\mathrm{n}}, \mathrm{f} \mathcal{\varkappa}_{\mathrm{n}+1}\right) \\
\leq \mathrm{p}\left(\mathrm{f} \mathcal{\varkappa}_{\mathrm{n}}, \mathrm{g} \mathcal{\varkappa}_{\mathrm{n}}\right)+\mathrm{p}\left(\mathrm{g} \mathcal{\varkappa}_{\mathrm{n}}, \mathrm{g} \mathcal{\varkappa}_{\mathrm{n}+1}\right)+\mathrm{p}\left(\mathrm{g} \mathcal{\varkappa}_{\mathrm{n}+1}, \mathrm{f} \mathcal{\varkappa}_{\mathrm{n}+1}\right)-\mathrm{p}\left(\mathrm{g} \mathcal{\varkappa}_{\mathrm{n}+1},\right. \\
\left.\mathrm{g} \mathcal{\varkappa}_{\mathrm{n}+1}\right) \\
\leq \mathrm{p}\left(\mathrm{f} \mathcal{\varkappa}_{\mathrm{n}}, \mathrm{g} \mathcal{\varkappa}_{\mathrm{n}}\right)+\mathrm{p}\left(\mathrm{g} \mathcal{\varkappa}_{\mathrm{n}}, \mathrm{g} \mathcal{\varkappa}_{\mathrm{n}+1}\right)+\mathrm{p}\left(\mathrm{g} \mathcal{\varkappa}_{\mathrm{n}+1}, \mathrm{f} \mathcal{\varkappa}_{\mathrm{n}+1}\right) .
\end{gathered}
$$

Now (6) becomes,

$$
\begin{gathered}
\mathrm{p}\left(\mathrm{g} \mathcal{H}_{\mathrm{n}}, \mathrm{g} \mathcal{\varkappa}_{\mathrm{n}+1}\right) \leq \alpha\left(\mathrm{p}\left(\mathrm{f} \mathcal{\varkappa}_{\mathrm{n}}, \mathrm{g} \mathcal{H}_{\mathrm{n}}\right)+\mathrm{p}\left(\mathrm{g} \mathcal{\varkappa}_{\mathrm{n}}, \mathrm{g} \mathcal{\varkappa}_{\mathrm{n}+1}\right)+\mathrm{p}\left(\mathrm{g} \mathcal{\varkappa}_{\mathrm{n}+1},\right.\right. \\
<\alpha\left(\frac{1}{n^{2}}+\frac{1}{(n+1)^{2}}\right)+\alpha \mathrm{p}\left(\mathrm{g} \mathcal{\varkappa}_{\mathrm{n}}, \mathrm{g} \mathcal{\varkappa}_{\mathrm{n}+1}\right)
\end{gathered}
$$


$\Rightarrow \quad \mathrm{p}\left(\mathrm{g} \mathcal{U}_{\mathrm{n}}, \mathrm{g} \mathcal{H}_{\mathrm{n}+1}\right)<\frac{\alpha}{(1-\alpha)}\left(\frac{1}{n^{2}}+\frac{1}{(n+1)^{2}}\right)$.

For $\mathrm{m} \leq n$, we obtain

$$
\begin{aligned}
& \mathrm{p}\left(\mathrm{g} \mathcal{H}_{\mathrm{m}}, \mathrm{g} \mathcal{\varkappa}_{\mathrm{n}}\right) \leq \mathrm{p}\left(\mathrm{g} \mathcal{\varkappa}_{\mathrm{m}}, \mathrm{g} \mathcal{\varkappa}_{\mathrm{m}+1}\right)+\mathrm{p}\left(\mathrm{g} \mathcal{\varkappa}_{\mathrm{m}+1}, \mathrm{~g} \mathcal{H}_{\mathrm{m}+2}\right)+\mathrm{p}\left(\mathrm{g} \mathcal{\varkappa}_{\mathrm{m}+2},\right. \\
& \left.\mathrm{g} \varkappa_{\mathrm{m}+3}\right)+\ldots \ldots \ldots+\mathrm{p}\left(\mathrm{g} \varkappa_{\mathrm{n}-1}, \mathrm{~g} \varkappa_{\mathrm{n}}\right) \\
& =\sum_{k=m}^{n-1} \mathrm{p}\left(\mathrm{g} \varkappa_{k}, \mathrm{~g} \varkappa_{k+1}\right) \\
& \leq \frac{\alpha}{(1-\alpha)} \sum_{k=m}^{n-1} \frac{1}{k^{2}} \text {. }
\end{aligned}
$$

By Cauchy criteria for the series, the series $\sum \frac{1}{n^{2}}$ and sequence of partial sums $\mathrm{Sn}=\sum_{k=1}^{n} \frac{1}{k^{2}}$ both converge together. Therefore, the sequence $\left\{\mathrm{g} \mathcal{\varkappa}_{n}\right\}$ is Cauchy and by Lemma, $\left\{\mathrm{f} \mathcal{\varkappa}_{n}\right\}$ is also Cauchy sequence. By hypothesis of $\mathrm{M}$, there exists 3 in $\mathrm{M}$ such that $\left\{\mathrm{g} \varkappa_{n}\right\}$ and $\left\{\mathrm{f} \varkappa_{n}\right\}$ both converge to 3 , since $\mathrm{p}\left(\mathrm{f} \mathcal{\varkappa}_{n}, \mathrm{~g} \varkappa_{n}\right) \rightarrow 0$ as $\mathrm{n} \rightarrow \infty$ and $\lim _{n \rightarrow \infty} \mathrm{p}\left(\mathrm{g} \mathcal{\varkappa}_{n}, 3\right)=\lim _{n \rightarrow \infty} \mathrm{p}\left(\mathrm{g} \mathcal{\varkappa}_{n}, \mathrm{~g} \mathcal{\varkappa}_{n}\right)=\mathrm{p}(3,3)=\lim _{n \rightarrow \infty} \mathrm{p}\left(\mathrm{f} \mathcal{\varkappa}_{n}\right.$, $\left.\mathrm{f} \mathcal{\varkappa}_{n}\right)=\lim _{n \rightarrow \infty} \mathrm{p}\left(\mathrm{f} \mathcal{\varkappa}_{n}, 3\right)$ but $\lim _{n \rightarrow \infty} \mathrm{p}\left(\mathrm{g} \mathcal{\varkappa}_{n}, \mathrm{~g} \mathcal{\varkappa}_{m}\right)=0$, therefore $p(3,3)=0$.

By G-continuity of $\mathrm{f}$ and using $(*), \mathrm{f}^{2} \varkappa_{\mathrm{n}}, \mathrm{fg} \mathcal{\varkappa}_{\mathrm{n}} \rightarrow \mathrm{f} \xi$.

Since $\mathrm{f}^{2} \mathcal{H}_{\mathrm{n}} \rightarrow \mathrm{f} \xi$ then by $(* *),\left(\mathrm{f}^{2} \mathcal{\varkappa}_{\mathrm{n}}, \mathrm{f} \xi\right),\left(\mathrm{f} \mathcal{H}_{\mathrm{n}}, \mathrm{f} \xi\right) \in \mathrm{E}(\tilde{G})$ and using (2.2) with $(* *)$, we get $\mathrm{p}\left(\mathrm{gf} \mathcal{\varkappa}_{\mathrm{n}}, \mathrm{g} \xi\right) \leq \alpha \mathrm{p}\left(\mathrm{f}^{2} \varkappa_{\mathrm{n}}\right.$, f3) which implies that as $\mathrm{n} \rightarrow \infty, \mathrm{gf} \mathcal{H}_{\mathrm{n}} \rightarrow \mathrm{g} 3$ and $\lim _{n \rightarrow \infty} \mathrm{p}\left(\mathrm{gf} \mathcal{\varkappa}_{\mathrm{n}}\right.$, $\mathrm{g} 3)=\mathrm{p}(\mathrm{g} 3, \mathrm{~g} 3)=0$.

Since $p\left(f g \mathcal{H}_{\mathrm{n}}, \mathrm{gf} \mathcal{\varkappa}_{\mathrm{n}}\right) \rightarrow 0$, we have $\mathrm{f} 3=\mathrm{g} 3$.

Now again by (5) and $(* *)$, we have

$$
\begin{aligned}
& \mathrm{p}\left(\mathrm{g} \mathcal{H}_{\mathrm{n}}, \mathrm{g} 3\right) \leq \alpha \mathrm{p}\left(\mathrm{f}_{\mathrm{n}}, \mathrm{f} 3\right) \\
& \Rightarrow \mathrm{p}(3, \mathrm{~g} 3) \leq \alpha \mathrm{p}\left(3, \mathrm{f}_{3}\right)=\alpha \mathrm{p}\left(3_{3}, \mathrm{~g} 3\right) \text { as } \mathrm{n} \rightarrow \infty . \\
& \Rightarrow(1-\alpha) \mathrm{p}(3, \mathrm{~g} 3) \leq 0 \\
& \Rightarrow \mathrm{f}_{3}=\mathrm{g}_{3}=3 .
\end{aligned}
$$

Hence, $f$ and $g$ have a unique common fixed point.

Example 2.6. Let $\mathrm{M}=[0,1]$ and define $\mathrm{p}: \mathrm{M} \times M \rightarrow R^{+}$ be a partial metric $\operatorname{such}$ thatp $(\varkappa, y)=\max \{\varkappa, y\}$ then $(\mathrm{M}$, p) is complete partial metric space. Let $\mathrm{G}$ be digraph and $\mathrm{E}(\mathrm{G})=\Delta \cup\{(\varkappa, y):(\varkappa, y) \in[0,1) \times[0,1)\}$. Also map $\varphi$ defined as $\varphi(\mathcal{\varkappa})=\mathcal{H}^{2}$.

Let $\mathrm{f}, \mathrm{g}: \mathrm{M} \rightarrow \mathrm{M}$ be defined by

$$
\mathrm{f} \mathcal{X}=\frac{2}{3} \mathcal{H} \text { and } \mathrm{g} \mathcal{X}=\left\{\begin{array}{c}
\frac{1}{3} \mathcal{H}^{2}, \mathcal{H} \neq 1 \\
\frac{1}{2}, \mathcal{\varkappa}=1
\end{array} \forall \varkappa \in M .\right.
$$

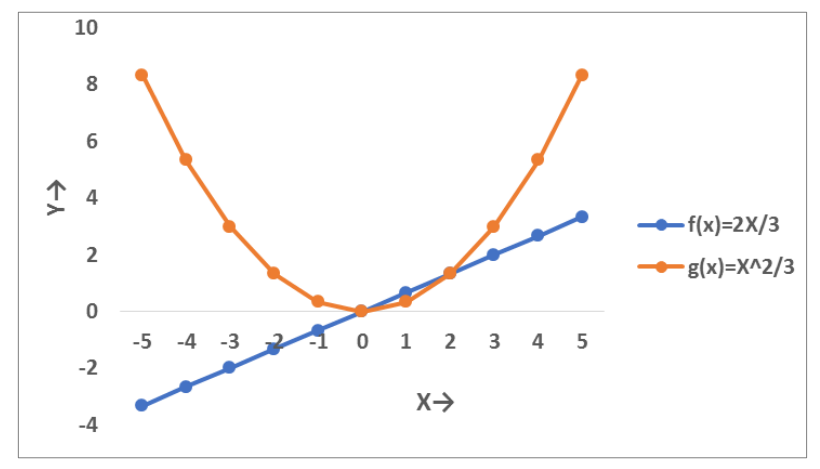

Figure 2. Maps $f$ and $g$ are $\varepsilon$-compatible
W.L.G choosing $\geq y$ and $\forall \varkappa, y \in \mathrm{E}(\tilde{G})$, we have

$$
\mathrm{p}(\mathrm{g} \mathcal{H}, \mathrm{g} y)=\max \left\{\frac{1}{3} \mathcal{\varkappa}^{2}, \frac{1}{3} y^{2}\right\}=\frac{1}{3} y^{2},
$$

$$
\mathrm{p}(\mathrm{f} \mathcal{\mathrm { f }} \mathrm{f})=\max \left\{\frac{2}{3} \mathcal{\varkappa}, \frac{2}{3} \mathrm{y}\right\}=\frac{2}{3} \mathcal{\varkappa} \text { and similarly }
$$

$$
\mathrm{p}(\mathrm{f} \mathcal{\mathrm { g }}, \mathrm{g} \mathrm{y})=\frac{2}{3} \mathcal{\varkappa}, \mathrm{p}(\mathrm{f} y, \mathrm{~g} \mathcal{H})=\frac{2}{3} y .
$$

(since $x, y \in[0,1)$ and $x \geq y \Rightarrow \varkappa \geq y \geq y^{2} \geq \varkappa^{2}$ )

If we take $\mathrm{a}=\frac{1}{4}, \mathrm{~b}=\frac{1}{4}$ and $\mathrm{c}=\frac{1}{8}$

then

$\mathrm{p}(\mathrm{g} \varkappa, \mathrm{g} y) \leq \mathrm{a} p(\mathrm{f} \mathcal{\mathrm { f }}, \mathrm{f} y)+\mathrm{b} \mathrm{p}(\mathrm{f} \mathcal{\mathrm { g }}, \mathrm{gy})+\mathrm{c} \mathrm{p}(\mathrm{fy}, \mathrm{g} \mathcal{H})$

i.e., $\frac{1}{3} y^{2} \leq \frac{1}{4}\left(\frac{2}{3} \varkappa\right)+\frac{1}{4}\left(\frac{2}{3} \varkappa\right)+\frac{1}{8}\left(\frac{2}{3} y\right)=\frac{1}{3}\left(\varkappa+\frac{y}{4}\right)$ which is true.

Also, $a+b+c \neq 1$ and $a+b+c<1$.

Now,

$$
\begin{gathered}
\mathrm{p}(\mathrm{f} \mathcal{\varkappa}, \mathrm{g} \mathcal{\varkappa})=\max \left\{\frac{2 \varkappa}{3}, \frac{\varkappa^{2}}{3}\right\}=\frac{2 \varkappa}{3} \\
\text { and } \mathrm{p}(\mathrm{fg} \mathcal{H}, \mathrm{gf} \mathcal{H})=\max \left\{\mathrm{f}\left(\frac{\mathcal{\varkappa}^{2}}{3}\right), \mathrm{g}\left(\frac{2 \varkappa}{3}\right)\right\} \\
=\max \left\{\frac{2}{3} \times \frac{\varkappa^{2}}{3}, \frac{1}{3} \times\left(\frac{2 \varkappa}{3}\right)^{2}\right\} \\
=\frac{2}{9} \max \left\{\mathcal{\varkappa}^{2}, \frac{2}{3} \varkappa^{2}\right\}=\frac{2}{9} \varkappa^{2} .
\end{gathered}
$$

Again, $\mathrm{p}(\mathrm{f} \mathcal{H}, \mathrm{g} \mathcal{H})<\varepsilon(n)$ if $\frac{2 \varkappa}{3}<\varepsilon(n)$ if $\mathcal{x}<\frac{3}{2} \varepsilon(n)$ implies $\mathrm{p}(\mathrm{fg} \mathcal{X}$, gf $\mathcal{H})<\varphi(\varepsilon(n))$ if $\frac{2}{9} \varkappa^{2}<\varepsilon^{2}(\mathrm{n})$ if $\varkappa<\frac{3}{2} \varepsilon(n)$.

Therefore, for each $\varepsilon(n)>0$ we can take element $\mathcal{H}_{\mathrm{n}}=$ $\frac{3}{4} \varepsilon(n)$ (you can also take another sequence but $\varkappa_{\mathrm{n}}<\frac{3}{2} \varepsilon(n)$ ) such that $\mathrm{p}(\mathrm{f} \mathcal{\mathrm { g }}, \mathrm{g} \mathcal{X})<\varepsilon(n)$ impliesp $(\mathrm{fg} \mathcal{X}, \mathrm{gf} \mathcal{H})<\varphi(\varepsilon(n))$, so $\mathrm{f}$ and $\mathrm{g}$ are $\varepsilon$-compatible and also $\left(\mathrm{f} \mathcal{H}_{\mathrm{n}}, \mathrm{f} \mathcal{H}_{\mathrm{n}+1}\right) \in \mathrm{E}(\tilde{G})$ and $\left(\mathrm{g} \varkappa_{\mathrm{n}}, \mathrm{g} \varkappa_{\mathrm{n}+1}\right) \in \mathrm{E}(\tilde{G})$. Consequently, it follows that property $(*)$ hold. Also, from the Figure 2., we can analyse that both mappings come very close to each other towards the point zero which provides atleast one element $\mathcal{H}$ in $\mathrm{M}$ for each $\varepsilon>0$ such that $\mathrm{p}(\mathrm{f} \mathcal{\mathrm { g }}, \mathrm{g} \mathcal{H})<\varepsilon$ implies $\mathrm{p}(\mathrm{fg} \mathcal{\mathrm { gf }} \mathcal{\mathrm { g }})$ $<\varphi(\varepsilon)$. Moreover, $\mathrm{f}$ is continuous which implies $\mathrm{G}$ is continuous, and the sequences converge to 1 and belong to $[0,1]$ such as $\left\{1-\frac{1}{n}\right\},\left\{1-\frac{1}{5 n}\right\},\left\{1-\frac{1}{n^{2}}\right\},\left\{1-\frac{1}{(2 n+1)^{2}}\right\}$ etc.

For any sequence, take $\varkappa_{\mathrm{n}}=1-\frac{1}{5 n}$ which converges to 1 with $\left(\varkappa_{\mathrm{n}}, \varkappa_{\mathrm{n}+1}\right) \in \mathrm{E}(\tilde{G})$ and $\mathrm{fx}_{\mathrm{n}}$ converges to $\frac{2}{3}=\mathrm{f} \mathcal{H}$ i.e, $\lim _{n \rightarrow \infty} \mathrm{p}\left(\mathrm{f} \mathcal{H}_{\mathrm{n}}, \mathrm{f} \mathcal{H}\right)=\mathrm{p}(\mathrm{f} \mathcal{f}, \mathrm{f} \mathcal{H})=\frac{2}{3}$, so condition $(* *)$ also hold and for any other sequences converging to any arbitrary element $(* *)$ also automatically hold.

Or in another way, we can also prove $f$ and $g$ are $\varepsilon$-compatible since $f(0)=g(0)=0$ and $f g(0)=g f(0)=0$ i.e, there exist a coincidence point and they commute at coincidence point.

Thus all the conditions of theorem 2.4 are satisfying and therefore, 0 is common fixed point of self-mappings $f$ and g.

Remark: In aboveexample, we can observe that the mappings $f$ and $g$ doesn't satisfy the contractive condition 
(2.1) on whole set $[0,1]$.

For example: If we take $\varkappa=0, y=1$ then $\mathrm{p}(\mathrm{g} \mathcal{x}, \mathrm{g} y)=2$ and $\mathrm{a} p(\mathrm{f} \mathcal{X}, \mathrm{f} y)+\mathrm{b} p(\mathrm{f} \mathcal{H}, \mathrm{g} y)+\mathrm{c} p(\mathrm{f} y, \mathrm{~g} \mathcal{X})=\mathrm{a}$ $\mathrm{p}\left(0, \frac{2}{3}\right)+\mathrm{b} p(0,2)+\mathrm{c} p\left(\frac{2}{3}, 0\right)=\frac{2}{3}(\mathrm{a}+\mathrm{c})+2 \mathrm{~b}$.

If $\mathrm{p}(\mathrm{g} \mathcal{H}, \mathrm{g} y) \leq \mathrm{a} p(\mathrm{f} \mathcal{H}, \mathrm{f} y)+b \mathrm{p}(\mathrm{f} \mathcal{H}, \mathrm{gy})+\mathrm{c} p(\mathrm{f} y, \mathrm{~g} \mathcal{X})$, that is,

$$
2 \leq \frac{2}{3}(a+c)+2 b
$$

where $a, b, c$ are in $R_{+}$such that $a+b+c \neq 1, a+b+c<1$.

Since $a+b+c<1$ implies that $(a+c)<(1-b)$

Now from (7), we have

$$
2 \leq \frac{2}{3}(a+c)+2 b<\frac{2}{3}(1-b)+2 b
$$

which implies $b>1$ that is not possible.

Thus, contractive condition doesn't hold for $\mathcal{\varkappa}=0, y=1$ and the given problem has no common fixed point by applying Theorem 2.1 with contractive condition on whole set. But this is possible if we restrict the contractive condition on some subset of $[0,1]$ and this is possible by inducing the graph.

Corollary 2.7. Let $\mathrm{f}$, g be $\varepsilon$-compatible mappings on a complete partial metric space (M, p) endowed with a graph $\mathrm{G}$ satisfies the condition

$\mathrm{p}(\mathrm{g} \mathcal{K}, \mathrm{gy}) \leq \mathrm{a} p(\mathrm{f} \mathcal{H}, \mathrm{fy})+\mathrm{b} p(\mathrm{f} \mathcal{H}, \mathrm{gy}), \forall(\mathcal{H}, \mathrm{y}) \in \mathrm{E}(\tilde{G})$

where $a, b$ are in $R_{+}$such that $a+b \neq 1, a+b<1$.

Assume that $f$ is $G$ - continuous with the following property:

(*) As $\mathrm{f}$ and $\mathrm{g}$ are $\varepsilon$ - compatible pair, so for every $\varepsilon(n)>0$, there exists an element $\mathcal{H}_{\mathrm{n}}$ in $\mathrm{M}$ such that $\mathrm{p}\left(\mathrm{f} \mathcal{H}_{\mathrm{n}}\right.$, $\left.\mathrm{g} \mathcal{H}_{\mathrm{n}}\right)<\varepsilon(n) \Rightarrow \mathrm{p}\left(\mathrm{fg} \mathcal{H}_{\mathrm{n}}, \mathrm{gf} \mathcal{H}_{\mathrm{n}}\right)<\varphi(\varepsilon(n))$ then $\left(\mathcal{H}_{\mathrm{n}}, \mathcal{H}_{\mathrm{n}+1}\right)$, $\left(\mathrm{f} \mathcal{H}_{\mathrm{n}}, \mathrm{f} \mathcal{H}_{\mathrm{n}+1}\right) \in \mathrm{E}(\tilde{G})$ and $\left(\mathrm{g} \mathcal{H}_{\mathrm{n}}, \mathrm{g} \mathcal{H}_{\mathrm{n}+1}\right) \epsilon \mathrm{E}(\tilde{G})(\varepsilon(n)$ depends on $\mathrm{n}$ or can say function of $\mathrm{n}$ and take less than one );

$(* *)$ If a sequence $\mathrm{f} \mathcal{H}_{\mathrm{n}} \rightarrow \mathcal{H}$ then $\left(\mathrm{f} \mathcal{H}_{\mathrm{n}}, \mathcal{H}\right) \in \mathrm{E}(\tilde{G})$ and $\left(\mathcal{H}_{\mathrm{n}}\right.$, $\varkappa) \epsilon \mathrm{E}(\tilde{G})$. Further $\mathrm{f}$ satisfies the condition $\operatorname{ifp}(\varkappa, \varkappa)=0$ then $\mathrm{p}(\mathrm{f} \mathcal{G}, \mathrm{f} \mathcal{H})=0$.

Then $\mathrm{f}$ and $\mathrm{g}$ have common fixed point. Moreover, if $\mathrm{u}, \mathrm{v}$ are fixed point of $\mathrm{f}$ and $\mathrm{g}$ with $(\mathrm{u}, \mathrm{v}) \epsilon \mathrm{E}(\widetilde{G})$ then $\mathrm{f}$ and $\mathrm{g}$ have unique common fixed point.

Proof: Put $\mathrm{c}=0$ in Theorem 2.4 then we obtain our required result.

Theorem 2.8. Let (M, p, G) be a complete partial metric space endowed with graph $\mathrm{G}$ and $\mathrm{f}$, g be $\varepsilon$-compatible mappings $\mathrm{M}$ which satisfies the condition

$$
\mathrm{p}(\mathrm{g} \varkappa, \mathrm{gy}) \leq \psi(\mathrm{m}(\varkappa, y)), \forall(\varkappa, y) \in \mathrm{E}(\tilde{G})
$$

where $\mathrm{m}(\mathcal{H}, y)=\max \left\{\mathrm{p}(\mathrm{f} \mathcal{H}, \mathrm{g} \mathcal{H}), \mathrm{p}(\mathrm{fy}, \mathrm{gy}), \frac{1}{4}\{\mathrm{p}(\mathrm{f} \mathcal{H}, \mathrm{gy})\right.$ $+p(f y, g \mathcal{K})\}\}$

Assume that $f$ is $G$ - continuous with the following property:

(*) As $\mathrm{f}$ and $\mathrm{g}$ are $\varepsilon$ - compatible pair, so for every $\varepsilon(n)>0$, there exists an element $\mathcal{\varkappa}_{\mathrm{n}}$ in M such that $\mathrm{p}\left(\mathrm{f} \mathcal{H}_{\mathrm{n}}\right.$, $\left.\mathrm{g} \mathcal{H}_{\mathrm{n}}\right)<\varepsilon(n) \Rightarrow \mathrm{p}\left(\mathrm{fg} \mathcal{H}_{\mathrm{n}}, \mathrm{gf} \mathcal{H}_{\mathrm{n}}\right)<\varphi(\varepsilon(n))$ then $\left(\mathcal{H}_{\mathrm{n}}, \mathcal{H}_{\mathrm{n}+1}\right)$, $\left(\mathrm{f} \mathcal{H}_{\mathrm{n}}, \mathrm{f} \mathcal{H}_{\mathrm{n}+1}\right) \in \mathrm{E}(\tilde{G})$ and $\left(\mathrm{g} \mathcal{H}_{\mathrm{n}}, \mathrm{g} \mathcal{U}_{\mathrm{n}+1}\right) \in \mathrm{E}(\tilde{G})(\varepsilon(n)$ depends on $\mathrm{n}$ or can say function of $\mathrm{n}$ and take less than one );

$(* *)$ If a sequence $\mathrm{f} \mathcal{H}_{\mathrm{n}} \rightarrow \mathcal{\varkappa}$ then $\left(\mathrm{f} \mathcal{H}_{\mathrm{n}}, \mathcal{\varkappa}\right) \in \mathrm{E}(\tilde{G})$ and $\left(\mathcal{H}_{\mathrm{n}}\right.$, $\varkappa) \epsilon \mathrm{E}(\tilde{G})$. Further $\mathrm{f}$ satisfies the condition if $\mathrm{p}(\mathcal{\varkappa}, \mathcal{\varkappa})=0$ then $\mathrm{p}(\mathrm{f} \mathcal{H}, \mathrm{f} \mathcal{H})=0$.

Then $\mathrm{f}$ and $\mathrm{g}$ have common fixed point. Moreover, if $\mathrm{u}, \mathrm{v}$ are fixed point of $\mathrm{f}$ and $\mathrm{g}$ with $(\mathrm{u}, \mathrm{v}) \epsilon \mathrm{E}(\tilde{G})$ then $\mathrm{f}$ and $\mathrm{g}$ have unique common fixed point.

Proof: Since $\mathrm{f}$ and $\mathrm{g}$ are $\varepsilon$-compatible, so their $\mathcal{\varepsilon}$-compatibility ensures that there exists a sequence $\left\{\mathcal{H}_{\mathrm{n}}\right\}$ in $\mathrm{M}$ such that for every $\mathrm{n} \in \mathrm{N}, \mathrm{p}\left(\mathrm{f} \varkappa_{\mathrm{n}}, \mathrm{g} \mathcal{\varkappa}_{\mathrm{n}}\right)<\frac{1}{n^{2}} \Rightarrow \mathrm{p}\left(\mathrm{fg} \mathcal{\varkappa}_{\mathrm{n}}\right.$, gf $\left.\mathcal{H}_{\mathrm{n}}\right)<\varphi\left(\frac{1}{n^{2}}\right)$.

Now, we have by using (9) and (*)

$$
\mathrm{p}\left(\mathrm{g} \mathcal{H}_{\mathrm{n}}, \mathrm{g} \mathcal{\varkappa}_{\mathrm{n}+1}\right) \leq \psi\left(\mathrm{m}\left(\mathcal{H}_{\mathrm{n}}, \mathcal{H}_{\mathrm{n}+1}\right)\right),
$$

where $\mathrm{m}\left(\mathcal{H}_{\mathrm{n}}, \mathcal{\varkappa}_{\mathrm{n}+1}\right)=\max \left\{\mathrm{p}\left(\mathrm{f} \mathcal{\varkappa}_{\mathrm{n}}, \mathrm{g} \mathcal{H}_{\mathrm{n}}\right), \mathrm{p}\left(\mathrm{f} \mathcal{\varkappa}_{\mathrm{n}+1}, \mathrm{~g} \mathcal{U}_{\mathrm{n}+1}\right)\right.$, $\left.\frac{1}{2}\left\{\mathrm{p}\left(\mathrm{f} \mathcal{H}_{\mathrm{n}}, \mathrm{g} \mathcal{H}_{\mathrm{n}+1}\right)+\mathrm{p}\left(\mathrm{f} \mathcal{\varkappa}_{\mathrm{n}+1}, \mathrm{~g} \mathcal{U}_{\mathrm{n}}\right)\right\}\right\}$.

There are three cases arises:

Case I: $\mathrm{m}\left(\mathcal{H}_{\mathrm{n}}, \mathcal{H}_{\mathrm{n}+1}\right)=\mathrm{p}\left(\mathrm{f} \mathcal{H}_{\mathrm{n}}, \mathrm{g} \mathcal{H}_{\mathrm{n}}\right)$ then $\mathrm{p}\left(\mathrm{g} \mathcal{H}_{\mathrm{n}}, \mathrm{g} \mathcal{H}_{\mathrm{n}+1}\right) \leq \psi\left(\mathrm{m}\left(\mathcal{H}_{\mathrm{n}}, \mathscr{H}_{\mathrm{n}+1}\right)\right)$ $\left.<\mathrm{m}\left(\mathcal{H}_{\mathrm{n}}, \mathcal{H}_{\mathrm{n}+1}\right)\right)=\mathrm{p}\left(\mathfrak{f}_{\mathrm{n}}, \mathrm{g} \mathcal{H}_{\mathrm{n}}\right) \leq \frac{1}{n^{2}}$.

(as $\psi$ is non- decreasing)

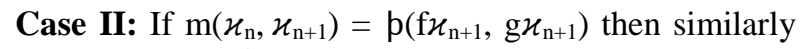
$\mathrm{p}\left(\mathrm{g} \varkappa_{\mathrm{n}}, \mathrm{g} \varkappa_{\mathrm{n}+1}\right) \leq \frac{1}{(n+1)^{2}}$.

Case III: If $\mathrm{m}\left(\varkappa_{\mathrm{n}}, \varkappa_{\mathrm{n}+1}\right)=\frac{1}{4}\left\{\mathrm{p}\left(\mathrm{f} \mathcal{\varkappa}_{\mathrm{n}}, \varkappa_{\mathrm{n}+1}\right)+\mathrm{p}\left(\mathrm{f} \varkappa_{\mathrm{n}+1}\right.\right.$, $\left.\left.\mathrm{g} \mathcal{U}_{\mathrm{n}}\right)\right\}$

$$
\begin{aligned}
& \leq \frac{1}{4}\left\{\mathrm{p}\left(\mathrm{f} \mathcal{\varkappa}_{\mathrm{n}}, \mathrm{g} \mathcal{\varkappa}_{\mathrm{n}}\right)+\mathrm{p}\left(\mathrm{g} \mathcal{\varkappa}_{\mathrm{n}}, \mathrm{g} \mathcal{\varkappa}_{\mathrm{n}+1}\right)+\mathrm{p}\left(\mathrm{f} \mathcal{\varkappa}_{\mathrm{n}+1}, \mathrm{~g} \mathcal{\varkappa}_{\mathrm{n}+1}\right)+\right. \\
& \left.\mathrm{p}\left(\mathrm{g} \mathcal{U}_{\mathrm{n}+1}, \mathrm{~g} \mathcal{U}_{\mathrm{n}}\right)-\mathrm{p}\left(\mathrm{g} \mathcal{H}_{\mathrm{n}}, \mathrm{g} \mathcal{\varkappa}_{\mathrm{n}}\right)-\mathrm{p}\left(\mathrm{g} \mathcal{\varkappa}_{\mathrm{n}+1}, \mathrm{~g} \mathcal{\varkappa}_{\mathrm{n}+1}\right)\right\} \\
& \leq \frac{1}{4}\left\{\mathrm{p}\left(\mathrm{f} \mathcal{H}_{\mathrm{n}}, \mathrm{g} \mathcal{H}_{\mathrm{n}}\right)+\mathrm{p}\left(\mathrm{g} \mathcal{H}_{\mathrm{n}}, \mathrm{g} \mathcal{U}_{\mathrm{n}+1}\right)+\mathrm{p}\left(\mathrm{f} \mathcal{H}_{\mathrm{n}+1}, \mathrm{~g} \mathcal{H}_{\mathrm{n}+1}\right)+\right. \\
& \left.\mathrm{p}\left(\mathrm{g} \mathcal{H}_{\mathrm{n}+1}, \mathrm{~g} \mathcal{U}_{\mathrm{n}}\right)\right\}
\end{aligned}
$$

$\leq \frac{1}{4}\left(\frac{1}{n^{2}}+\frac{1}{(n+1)^{2}}\right)+\frac{1}{2} \mathrm{p}\left(\mathrm{g} \mathcal{\varkappa}_{\mathrm{n}}, \mathrm{g} \mathcal{H}_{\mathrm{n}+1}\right)$.

Then

$\mathrm{p}\left(\mathrm{g} \mathcal{H}_{\mathrm{n}}, \mathrm{g} \mathcal{H}_{\mathrm{n}+1}\right) \leq \psi\left(\mathrm{m}\left(\mathcal{H}_{\mathrm{n}}, \mathcal{H}_{\mathrm{n}+1}\right)\right)<\mathrm{m}\left(\mathcal{H}_{\mathrm{n}}, \mathcal{U}_{\mathrm{n}+1}\right)$

$$
\leq \frac{1}{4}\left(\frac{1}{n^{2}}+\frac{1}{(n+1)^{2}}\right)+\frac{1}{2} \mathrm{p}\left(\mathrm{g} \mathcal{\varkappa}_{\mathrm{n}}, \mathrm{g} \mathcal{H}_{\mathrm{n}+1}\right)
$$

(since $\psi$ is non- decreasing)

$$
\begin{aligned}
& \text { i.e, } \frac{1}{2} \mathrm{p}\left(\mathrm{g} \mathcal{n}_{\mathrm{n}}, \mathrm{g} \mathcal{\varkappa}_{\mathrm{n}+1}\right) \leq \frac{1}{4}\left(\frac{1}{n^{2}}+\frac{1}{(n+1)^{2}}\right) \\
& \text { or } \mathrm{p}\left(\mathrm{g} \mathcal{\varkappa}_{\mathrm{n}}, \mathrm{g} \mathcal{\varkappa}_{\mathrm{n}+1}\right) \leq \frac{1}{2}\left(\frac{1}{n^{2}}+\frac{1}{(n+1)^{2}}\right) .
\end{aligned}
$$

From all possibility, we observe that for any $n \in N$,

$$
\mathrm{p}\left(\mathrm{g} \mathcal{\varkappa}_{\mathrm{n}}, \mathrm{g} \mathcal{\varkappa}_{\mathrm{n}+1}\right) \leq \frac{1}{n^{2}} .
$$

Now, for $\mathrm{m} \leq n$, we have

$$
\begin{gathered}
\mathrm{p}\left(\mathrm{g} \mathcal{\varkappa}_{\mathrm{m}}, \mathrm{g} \mathcal{\varkappa}_{\mathrm{n}}\right) \leq \mathrm{p}\left(\mathrm{g} \mathcal{\varkappa}_{\mathrm{m}}, \mathrm{g} \varkappa_{\mathrm{m}+1}\right)+\mathrm{p}\left(\mathrm{g} \mathcal{\varkappa}_{\mathrm{m}+1}, \mathrm{~g} \mathcal{\varkappa}_{\mathrm{m}+2}\right)+\mathrm{p}\left(\mathrm{g} \mathcal{\varkappa}_{\mathrm{m}+2},\right. \\
\left.\mathrm{g} \mathcal{\varkappa}_{\mathrm{m}+3}\right)+\ldots \ldots \ldots+\mathrm{p}\left(\mathrm{g} \mathcal{\varkappa}_{\mathrm{n}-1}, \mathrm{~g} \mathcal{\varkappa}_{\mathrm{n}}\right) \\
=\sum_{k=m}^{n-1} \mathrm{p}\left(\mathrm{g} \varkappa_{k}, \mathrm{~g} \varkappa_{k+1}\right) \\
\leq \sum_{k=m}^{n-1} \frac{1}{k^{2}} .
\end{gathered}
$$


Since the series $\sum \frac{1}{n^{2}}$ conveges, by cauchy criteria for the convergence of a series, the sequence $\left\{\mathrm{g} \mathcal{\varkappa}_{\mathrm{n}}\right\}$ is cauchyand by lemma, $\left\{\mathrm{f} \mathcal{H}_{\mathrm{n}}\right\}$ is also Cauchy. As $\mathrm{M}$ is complete, there exists $\mathrm{u} \in \mathrm{M}$ such that the sequences $\left\{\mathrm{g} \mathcal{H}_{\mathrm{n}}\right\}$ and $\left\{\mathrm{f} \mathcal{\varkappa}_{\mathrm{n}}\right\}$ converges to $\mathrm{u}$, since $\mathrm{p}\left(\mathrm{f} \mathcal{\varkappa}_{\mathrm{n}}, \mathrm{g} \mathcal{\varkappa}_{\mathrm{n}}\right) \rightarrow 0$ as $\mathrm{n} \rightarrow \infty$ and

$$
\begin{gathered}
\lim _{n \rightarrow \infty} \mathrm{p}\left(\mathrm{g} \mathcal{H}_{\mathrm{n}}, \mathrm{u}\right)=\lim _{n \rightarrow \infty} \mathrm{p}\left(\mathrm{g} \mathcal{H}_{\mathrm{n}}, \mathrm{g} \mathcal{U}_{\mathrm{n}}\right)=\mathrm{p}(\mathrm{u}, \mathrm{u})=\lim _{n \rightarrow \infty} \mathrm{p}\left(\mathrm{f} \mathcal{H}_{\mathrm{n}}, \mathrm{f} \mathcal{\varkappa}_{\mathrm{n}}\right) \\
=\lim _{n \rightarrow \infty} \mathrm{p}\left(\mathrm{f} \mathcal{H}_{\mathrm{n}}, \mathrm{u}\right)
\end{gathered}
$$

but

$\lim _{n \rightarrow \infty} \mathrm{p}\left(\mathrm{g} \mathcal{\varkappa}_{\mathrm{n}}, \mathrm{g} \mathcal{\varkappa}_{\mathrm{m}}\right)=0$, therefore $\mathrm{p}(\mathrm{u}, \mathrm{u})=0$ and by $(* *), \mathrm{p}(\mathrm{fu}$,

$$
\mathrm{fu})=0 .
$$

G-continuity of $\mathrm{f}$ and by the condition $(*), \mathrm{fg} \mathcal{H}_{\mathrm{n}}, \mathrm{f}^{2} \mathcal{\varkappa}_{\mathrm{n}} \rightarrow$ fu and $\mathrm{p}\left(\mathrm{fg} \mathcal{\varkappa}_{\mathrm{n}}, \mathrm{gf} \mathcal{\varkappa}_{\mathrm{n}}\right) \rightarrow 0$ as $\mathrm{n} \rightarrow \infty$ with $\mathrm{p}(\mathrm{fu}, \mathrm{fu})=0$ implies $\left\{\mathrm{gf} \mathcal{\varkappa}_{\mathrm{n}}\right\}$ converges to fu. Suppose that fu $\neq$ gu then $p(f u, g u)>0$.

Now, we have using (9) and $(* *)$

$$
\mathrm{p}\left(\mathrm{gf} \mathcal{\varkappa}_{\mathrm{n}}, \mathrm{gu}\right) \leq \psi\left(\mathrm{m}\left(\mathrm{f} \mathcal{H}_{\mathrm{n}}, \mathrm{u}\right) \leq \mathrm{m}\left(\mathrm{f}_{\mathcal{H}_{\mathrm{n}}}, \mathrm{u}\right)\right.
$$

where

$$
\begin{gathered}
\mathrm{m}\left(\mathrm{f} \mathcal{\varkappa}_{\mathrm{n}}, \mathrm{u}\right)=\max \left\{\mathrm{p}\left(\mathrm{ff} \mathcal{\varkappa}_{\mathrm{n}}, \mathrm{gf} \mathcal{\varkappa}_{\mathrm{n}}\right), \mathrm{p}(\mathrm{fu}, \mathrm{gu}), \frac{1}{4}\left\{\mathrm{p}\left(\mathrm{ff} \mathcal{\varkappa}_{\mathrm{n}}, \mathrm{gu}\right)\right.\right. \\
\left.\left.+\mathrm{p}\left(\mathrm{fu}, \mathrm{gf} \mathcal{\varkappa}_{\mathrm{n}}\right)\right\}\right\}
\end{gathered}
$$

Therefore, as $n \rightarrow \infty$

$\mathrm{p}(\mathrm{fu}, \mathrm{gu})<\mathrm{p}(\mathrm{fu}, \mathrm{gu})$ which is a contradiction.

Hence, $\mathrm{fu}=\mathrm{gu}$.

Finally, we show that $\mathrm{fu}=\mathrm{gu}=\mathrm{u}$.

Again, by (9)

$$
\mathrm{p}\left(\mathrm{g} \mathcal{H}_{\mathrm{n}}, \mathrm{gu}\right) \leq \psi\left(\mathrm{m}\left(\mathcal{H}_{\mathrm{n}}, \mathrm{u}\right)\right)<\mathrm{m}\left(\mathcal{H}_{\mathrm{n}}, \mathrm{u}\right)(10)
$$

where

$$
\begin{gathered}
\mathrm{m}\left(\mathcal{K}_{\mathrm{n}}, \mathrm{u}\right)=\max \left\{\mathrm{p}\left(\mathrm{f} \mathcal{H}_{\mathrm{n}}, \mathrm{g} \mathcal{H}_{\mathrm{n}}\right), \mathrm{p}(\mathrm{fu}, \mathrm{gu}), \frac{1}{4}\left\{\mathrm{p}\left(\mathrm{f} \mathcal{H}_{\mathrm{n}}, \mathrm{gu}\right)+\right.\right. \\
\left.\left.\mathrm{p}\left(\mathrm{fu}, \mathrm{g} \mathcal{\varkappa}_{\mathrm{n}}\right)\right\}\right\} \\
\text { and } \lim _{n \rightarrow \infty} \mathrm{m}\left(\mathcal{\varkappa}_{\mathrm{n}}, \mathrm{u}\right)=\frac{1}{2} \mathrm{p}(\mathrm{u}, \mathrm{gu}) .
\end{gathered}
$$

So (10) becomes

$$
\begin{aligned}
\mathrm{p}(\mathrm{u}, \mathrm{gu}) & <\frac{1}{2} \mathrm{p}(\mathrm{u}, \mathrm{gu}) \text { implies that } \mathrm{p}(\mathrm{u}, \mathrm{gu})=0 \\
& \Rightarrow \mathrm{gu}=\mathrm{u} \text { and also } \mathrm{fu}=\mathrm{gu}=\mathrm{u} .
\end{aligned}
$$

Now, we show that $\mathrm{u}$ is the unique fixed point. Let $\mathrm{v} \neq \mathrm{u}$ be a point in $\mathrm{M}$ such that $\mathrm{fv}=\mathrm{gv}=\mathrm{v}$ then by (9), we have

$$
\mathrm{p}(\mathrm{gu}, \mathrm{gv}) \leq \psi(\mathrm{m}(\mathrm{u}, \mathrm{v}))<\mathrm{m}(\mathrm{u}, \mathrm{v})
$$

where $m(u, v)=\max \left\{p(f u, g u), p(f v, g v), \frac{1}{4}\{p(f u, g v)+\right.$ $p(f v, g u)\}\}$

$$
\mathrm{p}(\mathrm{u}, \mathrm{v})<\frac{1}{2} \mathrm{p}(\mathrm{u}, \mathrm{v})
$$

implies that $\mathrm{p}(\mathrm{u}, \mathrm{v})=0$ i.e, $\mathrm{u}=\mathrm{v}$.

Hence $f$ and $g$ have a unique common fixed point.

\section{Conclusions}

In this paper, we presented some common fixed point theorems with graph for $\varepsilon$-compatible maps which doesn't involve the containment of the ranges for the given mappings. Generally, we see that common fixed point results for more than one mapping existing in the literature involve the suitable containment of the ranges for the given mappings which is a strong assumption in its own. In addition, we presented some examples to demonstrate the definitions and our results.

\section{REFERENCES}

[1] A. C. M. Ran and M. C. B. Reurings, A fixed point theorem in partially ordered sets and some applications to matrix equations, Proc. Amer. Math. Soc. 132, 1435-1443. MR2053350 (2005a:47112), 2004.

[2] F. Bojor, Fixed point of $\varphi$-contraction in metric spaces endowed with a graph. Mathematics and Computer Science Series, 37 (4), 85-92, 2010.

[3] F. Bojor, Fixed points of Kannan mapping in metric spaces endowed with a graph. An St. uni.Ovidius Constanta, 20 (1), 31- 40, 2012.

[4] G. Jungck and B. E. Rhoades, Fixed points for set valued functions without continuity. Indian J. Pure. Appl. Math., 29 , no. 3, 227-238, 1998.

[5] G. Jungck, Common fixed points for non-continuous map non self-maps non-metric spaces. Far East. J. Math. Sci., 4, 199-215, 1996.

[6] G. Jungck, Commuting mappings and fixed points. Amer. Math. Monthly. 83, 261-263, 1976.

[7] G. Jungck, Compatible mappings and common fixed points. Internat J. Math. Math. Sci.,9(4), 771-779, 1986.

[8] I. Beg, A. R. Butt and S. Radojevic, The contraction principle for set-valued mapping on metric space with a graph. Computers and mathematics with Application, 60, 1214-1219, 2010.

[9] J. Jachymski, The contraction principle for mappings on a metric space with a graph. American mathematical society, 136 (4), 1359-1373, 2007.

[10] M. Abbas and T. Nazir, Common fixed point of a power graphic contraction pair in partial metric spaces endowed with a graph. Fixed point theory and applications, 2013:20 doi:10.1186/1687, 2013.

[11] N.Hussain, Z. Kadelburg, S. Radenović\& F. A. Solamy, Comparison Functions and Fixed Point Results in Partial Metric Spaces, Abstract and Applied Analysis, Volume 2012, ID: 605781, pp-15, 2012.

[12] R.K. Sharma and S. Bharti, Semi Weakly Compatibility of Maps and Fixed Point Theorems in Fuzzy Metric Space, Pure Mathematical Sciences, Vol. 5, no. 1, 33-4711, 2016.

[13] S. Muralisankar, and K. Jeyabal, $\varepsilon$-Compatible maps and 
common fixed point theorems, KYUNGPOOK Math. J. 56, 807-817, 2016.

[14] S.G. Matthews, Partial metric topology. Ann New York Acad. Sci. 728. Proc. 8thSummar conferrence Topology and applications, 183-197, 1994.

[15] S.K. Mohanta, Common fixed points in b-metric spaces endowed with a graph. Mathematical Bechnk, 68(2), 140-154. 2016. 